\title{
On the energy of a flow arising in shape optimization
}

\author{
PIERRE CARDALIAGUET ${ }^{\dagger}$ \\ Laboratoire de Mathématiques (UMR 6205), Université de Bretagne Occidentale, \\ 6 Av. Le Gorgeu, BP 809, 29285 Brest, France \\ OLIVIER LEY \\ Laboratoire de Mathématiques et Physique Théorique (CNRS UMR 6083), \\ Fédération de Recherche Denis Poisson (FR 2964), \\ Université François Rabelais, Tours, Parc de Grandmont, 37200 Tours, France
}

[Received 10 July 2006 and in revised form 11 September 2007]

\begin{abstract}
In [8] we have defined a viscosity solution for the gradient flow of the exterior Bernoulli free boundary problem. We prove here that the associated energy is non-increasing along the flow. For this we build a discrete gradient flow in the flavour of Almgren, Taylor and Wang [2].
\end{abstract}

\section{Introduction}

In this paper we continue our investigation of a "gradient flow" for the Bernoulli free boundary problem initiated in [8]. The exterior Bernoulli free boundary problem is to minimize the capacity of a set under volume constraints. Using a Lagrange multiplier $\lambda>0$, this problem can be recast into the minimization with respect to the set $\Omega$ of the functional

$$
\mathcal{E}_{\lambda}(\Omega)=\operatorname{cap}_{S}(\Omega)+\lambda|\Omega|
$$

where $\operatorname{cap}_{S}(\Omega)$ denotes the capacity of the set $\Omega$ with respect to some fixed set $S$ and $|\Omega|$ denotes the volume of $\Omega$. The set $\Omega$ is constrained to satisfy the inclusion $S \subset \subset \Omega$. Notice that there is a "competition" between the two terms in the minimization: the capacity is non-increasing with respect to inclusion whereas the volume is non-decreasing.

Such a problem has quite a long history and we refer to the survey paper [12] for references and interpretations in physics. Our study is motivated by several papers in numerical analysis where discrete gradient flows are built via a level-set approach in order to solve free boundary and shape optimization problems: see [1] and the references therein for the recent advances in this area. In this framework, the exterior Bernoulli free boundary problem appears as a model problem in order to better understand this numerical approach. In this work, we prove that the energy $\mathcal{E}_{\lambda}$ is nonincreasing along the generalized flow we built in [8]. This question is certainly essential to better explain the numerical schemes of [1].

Let us now go further into the description of the gradient flow for $\mathcal{E}:=\mathcal{E}_{1}$ (we work here in the case $\lambda=1$ for simplicity of notation). The energy $\mathcal{E}$ being defined on sets, a gradient flow for $\mathcal{E}$

\footnotetext{
${ }^{\dagger}$ E-mail: Pierre.Cardaliaguet@univ-brest.fr

‡E-mail: ley@1mpt.univ-tours.fr
} 
is a family $(\Omega(t))_{t} \geqslant 0$ of sets evolving with a normal velocity which "instantaneously decreases the energy the most". For the Bernoulli problem, the corresponding evolution law is given by

$$
V_{t, x}=h(x, \Omega(t)):=-1+\bar{h}(x, \Omega(t)) \quad \text { for all } t \geqslant 0, x \in \partial \Omega(t) .
$$

In the above equation, $V_{t, x}$ is the normal velocity of the set $\Omega(t)$ at the point $x$ at time $t$ and $\bar{h}(x, \Omega)$ is a non-local term of Hele-Shaw type, given, for any set $\Omega$ with smooth boundary, by

$$
\bar{h}(x, \Omega)=|\nabla u(x)|^{2},
$$

where $u: \Omega \rightarrow \mathbb{R}$ is the capacity potential of $\Omega$ with respect to $S$, i.e., the solution of the partial differential equation

$$
\begin{cases}-\Delta u=0 & \text { in } \Omega \backslash S, \\ u=1 & \text { on } \partial S, \\ u=0 & \text { on } \partial \Omega .\end{cases}
$$

The set $S$ is a fixed source and we always assume above that $S$ is smooth and $S \subset \subset \Omega(t)$. Let us underline that $h(x, \Omega)$ is well defined as soon as $\Omega$ has "smooth" (say $\mathcal{C}^{2}$ ) boundary and that $S \subset \subset \Omega$.

The reason why a smooth solution $(\Omega(t))$ of the geometric equation (1) can be considered as a gradient flow of the energy

$$
\mathcal{E}(\Omega)=|\Omega|+\operatorname{cap}_{S}(\Omega)
$$

is the following: from the Hadamard formula we have

$$
\frac{d}{d t} \mathcal{E}(\Omega(t))=\int_{\partial \Omega(t)}\left(1-|\nabla u|^{2}\right) V_{t, x}=-\int_{\partial \Omega(t)}\left(-1+|\nabla u|^{2}\right)^{2} \leqslant 0 .
$$

Hence the choice of $V_{t, x}=h(x, \Omega(t))$ in (1) appears to be the one which decreases the energy $\mathcal{E}$ the most. In order to minimize the energy $\mathcal{E}$, it is therefore very natural to follow the gradient flow (1). This is precisely what is done numerically in [1].

In general the geometric flow (1) does not have classical solutions. In order to define the flow after the onset of singularities, we have introduced in [8] a notion of generalized (viscosity) solution and investigated its existence as well as uniqueness. In order to prove that the energy is nonincreasing along the generalized flow, we face a main difficulty: energy estimates are hard to derive from the notion of viscosity solutions. Indeed, this latter notion is defined through a comparison principle, which has very little to do with the energy associated to the flow. To the best of our knowledge, such a question has only been settled for the mean curvature motion (MCM for short), which corresponds (at least formally) to the gradient flow of the perimeter. There are two proofs of the fact that the perimeter of the viscosity solution to the mean curvature flow decreases: the first one is due to Evans and Spruck in their seminal papers [10, 11]; it is based on a regularized version of the level set formulation for the flow and is probably specific to local evolution equations. The other proof is due to Chambolle [9]. Its starting point is the fundamental construction of Almgren, Taylor and Wang [2] who built generalized solutions of the MCM in a variational way as limits of a "discrete gradient flow" for the perimeter (the so-called minimizing movements; see also Ambrosio [5]). The key argument of Chambolle's paper [9] is that Almgren, Taylor and Wang's generalized solutions coincide with the viscosity solutions, at least for a large class of initial sets. Hence the 
energy estimate available from [2]—which allows comparing the energy of the evolving set with the energy of the initial position — can also be applied to the viscosity solution. Since the viscosity solution enjoys a semigroup property, one can conclude that the energy is decreasing along the flow.

To prove that the energy $\mathcal{E}$ is decreasing along our viscosity solutions of (11), we borrow several ideas from Almgren, Taylor and Wang [2] and Chambolle [9]. As in [2] for the MCM, we start with the construction of a discrete gradient flow $\left(\Omega_{n}^{h}\right)$ for the energy $\mathcal{E}$ : namely $\Omega_{n+1}^{h}$ is obtained from $\Omega_{n}^{h}$ as a minimizer of a functional $J_{h}\left(\Omega_{n}^{h}, \cdot\right)$ which is equal to $\mathcal{E}$ plus a penalizing term. The penalizing term—which depends on the time step $h$-prevents the minimizing set $\Omega_{n+1}^{h}$ from being too far from $\Omega_{n}^{h}$. Then, as in Chambolle [9], we prove that the limits of these discrete gradient flows converge to the viscosity solution of our equation (1) as the time step $h$ goes to 0 . In [9], this convergence is proved by using the convexity of the equivalent of our functional $J_{h}\left(\Omega_{h}^{n}, \cdot\right)$ for the MCM. Here we use instead directly a weak form of the Euler equation for minimizers of $J_{h}\left(\Omega_{h}^{n}, \cdot\right)$ as described by Alt and Caffarelli [3] for the Bernoulli problem. We then conclude that the energy of the flow is non-increasing.

The paper is organized in the following way. In Section 2 we recall the construction of [8] for the viscosity solutions of (1). Section 3 is devoted to suitable generalizations of the capacity and capacity potential needed for our estimates. In Section 4 we introduce the functional $J_{h}$ and build the discrete motions, the limits of which are discussed in Section 5. The fact that the energy is non-increasing along the flow is finally proved in Section 6

\section{Definitions and notations for the generalized flow}

Let us first fix some basic notations: if $A, B$ are subsets of $\mathbb{R}^{N}$, then $A \subset \subset B$ means that the closure $\bar{A}$ of $A$ is a compact subset which satisfies $\bar{A} \subset \operatorname{int}(B)$, where $\operatorname{int}(B)$ is the interior of $B$. We set

$$
\mathcal{D}=\left\{K \subset \subset \mathbb{R}^{N}: S \subset \subset K\right\} .
$$

Throughout the paper $|\cdot|$ denotes the euclidean norm (in $\mathbb{R}^{N}$ or $\mathbb{R}^{N+1}$, depending on the context) and $B(x, R)$ denotes the open ball centered at $x$ and of radius $R$. If $E$ is a measurable subset of $\mathbb{R}^{N}$, we also denote by $|E|$ the Lebesgue measure of $E$. If $K$ is a subset of $\mathbb{R}^{N}$ and $x \in \mathbb{R}^{N}$, then $d_{K}(x)$ denotes the usual distance from $x$ to $K: d_{K}(x)=\inf _{y \in K}|y-x|$. The signed distance $d_{K}^{s}$ to $K$ is defined by

$$
d_{K}^{s}(x)= \begin{cases}d_{K}(x) & \text { if } x \notin K, \\ -d_{\partial K}(x) & \text { if } x \in K,\end{cases}
$$

where $\partial K=\bar{K} \backslash \operatorname{int}(K)$ is the boundary of $K$.

Here and throughout the paper, we assume that

$S$ is the closure of an open, nonempty, bounded subset of $\mathbb{R}^{N}$ with a $\mathcal{C}^{2}$ boundary.

The generalized solution of the front propagation problem (1) is defined through its graph: if $(\Omega(t))_{t \geqslant 0}$ is family of evolving sets, then its graph is the subset of $\mathbb{R}^{+} \times \mathbb{R}^{N}$ defined by

$$
\mathcal{K}=\left\{(t, x) \in \mathbb{R}^{+} \times \mathbb{R}^{N}: x \in \Omega(t)\right\} .
$$

We denote by $(t, x)$ an element of such a set, where $t \in \mathbb{R}^{+}$denotes the time and $x \in \mathbb{R}^{N}$ is the space coordinate. We set

$$
\mathcal{K}(t)=\left\{x \in \mathbb{R}^{N}:(t, x) \in \mathcal{K}\right\} .
$$


The closure of the set $\mathcal{K}$ in $\mathbb{R}^{N+1}$ is denoted by $\overline{\mathcal{K}}$. The closure of the complement of $\mathcal{K}$ is denoted $\widehat{\mathcal{K}}$ :

$$
\widehat{\mathcal{K}}=\overline{\left(\mathbb{R}^{+} \times \mathbb{R}^{N}\right) \backslash \mathcal{K}},
$$

and we set

$$
\widehat{\mathcal{K}}(t)=\left\{x \in \mathbb{R}^{N} \mid(t, x) \in \widehat{\mathcal{K}}\right\} .
$$

We use here repeatedly the terminology of [6, 7, 8]:

- A tube $\mathcal{K}$ is a subset of $\mathbb{R}^{+} \times \mathbb{R}^{N}$ such that $\overline{\mathcal{K}} \cap\left([0, t] \times \mathbb{R}^{N}\right)$ is a compact subset of $\mathbb{R}^{N+1}$ for any $t \geqslant 0$.

- A tube $\mathcal{K}$ is left lower semicontinuous if

$$
\forall t>0, \forall x \in \mathcal{K}(t), \forall t_{n} \rightarrow t^{-}, \exists x_{n} \in \mathcal{K}\left(t_{n}\right), \quad x_{n} \rightarrow x .
$$

- If $s=1,2$ or $(1,1)$, then a $\mathcal{C}^{s}$ tube $\mathcal{K}$ on some open interval $I$ of $\mathbb{R}^{+}$is a tube such that the relative boundary of $\mathcal{K}$ in $I \times \mathbb{R}^{N}$ is at least $\mathcal{C}^{s}$ regular.

- A regular tube $\mathcal{K}_{r}$ on some open interval $I$ of $\mathbb{R}^{+}$is a tube with non-empty interior such that the relative boundary of $\mathcal{K}_{r}$ in $I \times \mathbb{R}^{N}$ is at least $\mathcal{C}^{1}$ regular, and at any point $(t, x)$ of this boundary, the outward normal $\left(v_{t}, v_{x}\right)$ to $\mathcal{K}_{r}$ at $(t, x)$ satisfies $v_{x} \neq 0$. In this case, the normal velocity $V_{(t, x)}^{\mathcal{K}_{r}}$ at $(t, x) \in \partial \mathcal{K}_{r}$ is defined by

$$
V_{(t, x)}^{\mathcal{K}_{r}}=-\frac{v_{t}}{\left|v_{x}\right|}
$$

where $\left(v_{t}, v_{x}\right)$ is the outward normal to $\mathcal{K}_{r}$ at $(t, x)$.

- A $\mathcal{C}^{1}$ regular tube $\mathcal{K}_{r}$ on some open interval $I$ of $\mathbb{R}^{+}$is externally tangent to a tube $\mathcal{K}$ at $(t, x) \in \mathcal{K}$ if $t \in I$ and

$$
\left(I \times \mathbb{R}^{N}\right) \cap \mathcal{K} \subset \mathcal{K}_{r} \quad \text { and } \quad(t, x) \in \partial \mathcal{K}_{r} .
$$

It is internally tangent to $\mathcal{K}$ at $(t, x) \in \widehat{\mathcal{K}}$ if $t \in I$ and

$$
\left(I \times \mathbb{R}^{N}\right) \cap \mathcal{K}_{r} \subset \mathcal{K} \quad \text { and } \quad(t, x) \in \partial \mathcal{K}_{r} .
$$

- We say that a sequence of $\mathcal{C}^{1,1}$ tubes $\left(\mathcal{K}_{n}\right)$ converges to some $\mathcal{C}^{1,1}$ tube $\mathcal{K}$ in some open interval I in the $\mathcal{C}^{1, \mathrm{~b}}$ sense if $\left(\mathcal{K}_{n}\right)$ converges to $\mathcal{K}$ and $\left(\partial \mathcal{K}_{n}\right)$ converges to $\partial \mathcal{K}$ in the Hausdorff distance, and if there is an open neighborhood $\mathcal{O}$ of the relative boundary of $\mathcal{K}$ in $I \times \mathbb{R}^{N}$ such that, if $d_{\mathcal{K}}^{s}$ (respectively $d_{\mathcal{K}_{n}}^{s}$ ) is the signed distance to $\mathcal{K}$ (respectively to $\mathcal{K}_{n}$ ), then $\left(d_{\mathcal{K}_{n}}^{s}\right)$ and $\left(\nabla d_{\mathcal{K}_{n}}^{s}\right)$ converge uniformly to $d_{\mathcal{K}}^{S}$ and $D \mathbf{d}_{\mathcal{K}}$ on $\mathcal{O}$ and $\left\|D^{2} d_{\mathcal{K}_{n}}^{s}\right\|_{\infty}$ is uniformly bounded on $\mathcal{O}$.

We are now ready to define the generalized solutions of (1):

Definition 2.1 Let $\mathcal{K}$ be a tube and $K_{0} \in \mathcal{D}$ be an initial set.

1. $\mathcal{K}$ is a viscosity subsolution to the front propagation problem 11 if $\mathcal{K}$ is left lower semicontinuous and $\mathcal{K}(t) \in \mathcal{D}$ for any $t$, and, for any $\mathcal{C}^{2}$ regular tube $\mathcal{K}_{r}$ externally tangent to $\mathcal{K}$ at some point $(t, x)$, with $\mathcal{K}_{r}(t) \in \mathcal{D}$ and $t>0$, we have

$$
V_{(t, x)}^{\mathcal{K}_{r}} \leqslant h\left(x, \mathcal{K}_{r}(t)\right)
$$

where $V_{(t, x)}^{\mathcal{K}_{r}}$ is the normal velocity of $\mathcal{K}_{r}$ at $(t, x)$. 
We say that $\mathcal{K}$ is a subsolution to the front propagation problem with initial position $K_{0}$ if $\mathcal{K}$ is a subsolution and $\overline{\mathcal{K}}(0) \subset \overline{K_{0}}$.

2. $\mathcal{K}$ is a viscosity supersolution to the front propagation problem if $\widehat{\mathcal{K}}$ left lower semicontinuous and $\mathcal{K}(t) \subset \mathcal{D}$ for any $t$, and, for any $\mathcal{C}^{2}$ regular tube $\mathcal{K}_{r}$ internally tangent to $\mathcal{K}$ at some point $(t, x)$, with $\mathcal{K}_{r}(t) \in \mathcal{D}$ and $t>0$, we have

$$
V_{(t, x)}^{\mathcal{K}_{r}} \geqslant h\left(x, \mathcal{K}_{r}(t)\right) .
$$

We say that $\mathcal{K}$ is a supersolution to the front propagation problem with initial position $K_{0}$ if $\mathcal{K}$ is a supersolution and $\widehat{\mathcal{K}}(0) \subset \overline{\mathbb{R}^{N} \backslash K_{0}}$.

3. Finally, we say that a tube $\mathcal{K}$ is a viscosity solution to the front propagation problem (with initial position $K_{0}$ ) if $\mathcal{K}$ is a sub- and a supersolution to the front propagation problem (with initial position $K_{0}$ ).

In [8] we have proved that for any initial position there is a maximal solution, with a closed graph, which contains any subsolution of the problem, as well as a minimal solution, which has an open graph, and is contained in any supersolution of the problem.

\section{Capacity and capacity potential}

Let $\Omega$ be an open bounded subset of $\mathbb{R}^{N}$. We denote by $\mathcal{C}_{c}^{\infty}(\Omega)$ the set of smooth functions with compact support in $\Omega$, and by $H_{0}^{1}(\Omega)$ its closure in the $H^{1}$ norm. By convention, if $u \in H_{0}^{1}(\Omega)$, then we extend $u$ by setting $u=0$ on $\mathbb{R}^{N} \backslash \Omega$. Let $S$ be as in $(6$. For an open bounded subset $\Omega$ of $\mathbb{R}^{N}$ such that $S \subset \subset \Omega$, the capacity of $\Omega$ with respect to $S$ is defined by

$$
\operatorname{cap}_{S}(\Omega)=\inf \left\{\int_{\Omega \backslash S}|\nabla \phi|^{2}: \phi \in \mathcal{C}_{c}^{\infty}(\Omega), \phi=1 \text { on } S\right\} .
$$

Since $S$ is a fixed set in what follows, we will write cap $(\Omega)$ instead of $\operatorname{cap}_{S}(\Omega)$.

Obviously $\operatorname{cap}(\Omega)$ is non-increasing with respect to $\Omega$ (for inclusion). Note that

$$
\operatorname{cap}(\Omega)=\inf \left\{\int_{\Omega \backslash S}|\nabla v|^{2}: v \in H_{0}^{1}(\Omega), v=1 \text { on } S\right\}
$$

and the infimum is achieved for a unique $u \in H_{0}^{1}(\Omega)$, called the capacity potential of $\Omega$ with respect to $S$, such that $u=1$ on $S$, and $u$ is harmonic in $\Omega \backslash S$. If $\Omega$ has a $\mathcal{C}^{1,1}$ boundary, then it is known that the infimum is achieved by some $u \in \mathcal{C}^{2}(\Omega \backslash S) \cap \mathcal{C}^{1}(\overline{\Omega \backslash S})$ which is a classical solution to 3 .

For any set $E$ (not necessarily open) such that $S \subset \subset E$, we define a generalized capacity by

$$
\operatorname{cap}(\bar{E})=\sup \{\operatorname{cap}(\Omega): E \subset \subset \Omega, \Omega \text { open and bounded }\} .
$$

With this definition, $\operatorname{cap}(\bar{E})$ is non-increasing with respect to $E$. Notice that this notion of capacity does not take into account "thin closed sets" in the sense that, if $\bar{F}=\bar{E}$, then $\operatorname{cap}(\bar{E})=\operatorname{cap}(\bar{F})$ even when $|E \backslash F| \neq 0$. By construction, if $E$ is open, then

$$
\operatorname{cap}(\bar{E}) \leqslant \operatorname{cap}(E),
$$

but equality does not hold in general. Nevertheless, there is equality if the boundary of the set is regular enough: 
LEMMA 3.1 If $\Omega$ is an open bounded subset of $\mathbb{R}^{N}$, with $S \subset \subset \Omega$ and with a $\mathcal{C}^{1,1}$ boundary, then $\operatorname{cap}(\Omega)=\operatorname{cap}(\bar{\Omega})$.

Proof. We have to prove that $\operatorname{cap}(\bar{\Omega}) \geqslant \operatorname{cap}(\Omega)$. It is enough to show that, if

$$
\Omega_{n}=\left\{y \in \mathbb{R}^{N}: d_{\Omega}(y)<1 / n\right\}
$$

then $\operatorname{cap}\left(\Omega_{n}\right) \rightarrow \operatorname{cap}(\Omega)$ as $n \rightarrow+\infty$. Indeed, for $n$ large enough, $\Omega_{n}$ also has a $\mathcal{C}^{1,1}$ boundary. Then from classical regularity arguments, the harmonic potential $u_{n}$ to $\Omega_{n}$ converges to the capacity potential $u$ of $\Omega$ in the $\mathcal{C}^{1, \alpha}$ norm, where $\alpha \in(0,1)$, whence the result.

LEMMA 3.2 Let $E_{n}$ be a bounded sequence of subsets of $\mathbb{R}^{N}$, for which there exists some $r>0$ with $S_{r} \subset E_{n}$ for any $n$, where

$$
S_{r}=\left\{y \in \mathbb{R}^{N}: d_{S}(y) \leqslant r\right\}
$$

Denote by $K$ the Kuratowski upper limit of the $\left(E_{n}\right)$, that is,

$$
K=\left\{x \in \mathbb{R}^{N}: \liminf _{n} d_{E_{n}}(x)=0\right\} .
$$

Then

$$
\liminf _{n} \operatorname{cap}\left(\bar{E}_{n}\right) \geqslant \operatorname{cap}(\bar{K}) \text {. }
$$

Proof. Let $\Omega$ be any open bounded set such that $K \subset \subset \Omega$. Since $\left(E_{n}\right)$ is bounded and has $K$ as upper limit, the inclusion $E_{n} \subset \Omega$ holds for $n$ large enough. Hence $\operatorname{cap}\left(\bar{E}_{n}\right) \geqslant \operatorname{cap}(\Omega)$ for every $n$. Therefore

$$
\liminf _{n} \operatorname{cap}\left(\bar{E}_{n}\right) \geqslant \operatorname{cap}(\Omega) \text {. }
$$

The open set $\Omega$ being arbitrary, the desired conclusion holds.

Let $\Omega$ be an open bounded subset of $\mathbb{R}^{N}$ with $S \subset \subset \Omega$. We denote by $H_{0}^{1}(\bar{\Omega})$ the intersection of the spaces $H_{0}^{1}\left(\Omega_{n}\right)$ where $\left(\Omega_{n}\right)$ is a decreasing sequence of open bounded sets such that $\Omega \subset \subset \Omega_{n}$ and $\bar{\Omega}=\bigcap_{n} \Omega_{n}$. One easily checks that $H_{0}^{1}(\bar{\Omega})$ does not depend on the sequence $\left(\Omega_{n}\right)$.

LEMMA 3.3 Assume that $|\partial \Omega|=0$. Then

$$
\operatorname{cap}(\bar{\Omega})=\inf \left\{\int_{\Omega \backslash S}|\nabla v|^{2}: v \in H_{0}^{1}(\bar{\Omega}), v=1 \text { on } S\right\},
$$

and there is a unique $u \in H_{0}^{1}(\bar{\Omega})$ such that

$$
u=1 \text { on } S \quad \text { and } \quad \int_{\mathbb{R}^{N} \backslash S}|\nabla u|^{2}=\int_{\Omega \backslash S}|\nabla u|^{2}=\operatorname{cap}(\bar{\Omega}) .
$$

Moreover, $u$ is harmonic in $\Omega \backslash S$ and $|\{u>0\} \backslash \Omega|=0$.

DEFINITION 3.4 Such a function $u$ is called the capacity potential of $\bar{\Omega}$ with respect to $S$.

REMARK 3.1 1. If $\partial \Omega$ is $\mathcal{C}^{1,1}$, then the capacity potential $u$ of $\bar{\Omega}$ with respect to $S$ is the (classical) solution of (3) and is equal to the (classical) capacity potential (7) of $\Omega$. 
2. In what follows, we study the energy of subsets $\Omega \supset S$ which is defined as the sum of the capacity and the volume of $\Omega$ with respect to $S$ (see (4)). This energy is well-defined for bounded sets $\Omega \supset \supset$. That is why we assumed all the sets to be bounded. But let us mention that all classical results of this section hold upon replacing $\Omega, S$ bounded by $\Omega \backslash S$ bounded. We need this generalization in the proof of Lemma 4.5

Proof of Lemma 3.3. The proof is easily obtained by approximation. By construction of $\operatorname{cap}(\bar{\Omega})$, we can find a decreasing sequence of open bounded sets $\Omega_{n}$ such that

$$
\Omega \subset \subset \Omega_{n}, \quad \bigcap_{n} \Omega_{n}=\bar{\Omega} \text { and } \operatorname{cap}(\bar{\Omega})=\lim _{n} \operatorname{cap}\left(\Omega_{n}\right) .
$$

Let $u_{n}$ be the (classical) capacity potential of $\Omega_{n}$. From the maximum principle, the sequence $\left(u_{n}\right)$ is decreasing, and converges to some $u$ which is non-negative with support in $\bar{\Omega}$ and equals 1 on $S$. In particular, $\{u>0\} \subset \Omega$ a.e. since $|\partial \Omega|=0$. Furthermore, by a classical stability result, $u$ is harmonic in $\Omega$ because so are the $u_{n}$. Since we can find a smooth function $\phi$ with compact support in $\Omega$ such that $\phi=1$ on $S$, we have

$$
\int_{\Omega_{n} \backslash S}\left|\nabla u_{n}\right|^{2} \leqslant \int_{\Omega_{n} \backslash S}|\nabla \phi|^{2}=\int_{\Omega \backslash S}|\nabla \phi|^{2},
$$

which proves that $\left(u_{n}\right)$ is bounded in $H^{1}\left(\mathbb{R}^{N}\right)$. Thus the limit $u$ belongs to $H^{1}\left(\mathbb{R}^{N}\right)$. Since $u_{n} \in$ $H_{0}^{1}\left(\Omega_{n}\right)$ with $H_{0}^{1}\left(\Omega_{n+1}\right) \subset H_{0}^{1}\left(\Omega_{n}\right), u$ belongs to $H_{0}^{1}\left(\Omega_{n}\right)$ for any $n$. Therefore $u \in H_{0}^{1}(\bar{\Omega})$. In particular, the support of $u$ lies in $\bar{\Omega}=\Omega$ a.e. So we have

$$
\begin{aligned}
\operatorname{cap}(\bar{\Omega}) & =\lim _{n} \operatorname{cap}\left(\Omega_{n}\right)=\lim _{n} \int_{\Omega_{n} \backslash S}\left|\nabla u_{n}\right|^{2} \\
& =\liminf _{n} \int_{\mathbb{R}^{N} \backslash S}\left|\nabla u_{n}\right|^{2} \geqslant \int_{\mathbb{R}^{N} \backslash S}|\nabla u|^{2}=\int_{\Omega \backslash S}|\nabla u|^{2} .
\end{aligned}
$$

For every $n$,

$$
\begin{aligned}
\operatorname{cap}\left(\Omega_{n}\right)=\int_{\Omega_{n} \backslash S}\left|\nabla u_{n}\right|^{2} & =\inf \left\{\int_{\Omega_{n} \backslash S}|\nabla v|^{2}: v \in H_{0}^{1}\left(\Omega_{n}\right), v=1 \text { on } S\right\} \\
& \leqslant \inf \left\{\int_{\Omega \backslash S}|\nabla v|^{2}: v \in H_{0}^{1}(\bar{\Omega}), v=1 \text { on } S\right\},
\end{aligned}
$$

since $H_{0}^{1}(\bar{\Omega}) \subset H_{0}^{1}\left(\Omega_{n}\right)$. Letting $n$ go to infinity, we obtain

$$
\operatorname{cap}(\bar{\Omega}) \leqslant \inf \left\{\int_{\Omega \backslash S}|\nabla v|^{2}: v \in H_{0}^{1}(\bar{\Omega}), v=1 \text { on } S\right\} .
$$

From (9), we get equality in the above inequality and the fact that $u$ is optimal.

\section{The discrete motions}

Let us fix $h>0$ which has to be understood as a time step. Let us recall that $S$ is the closure of an open bounded subset of $\mathbb{R}^{N}$ with $\mathcal{C}^{2}$ boundary. We introduce the function space

$$
E(S):=\left\{u \in H^{1}\left(\mathbb{R}^{N}\right) \cap L^{\infty}\left(\mathbb{R}^{N}\right): u=1 \text { on } S \text { and } u \text { has compact support }\right\} .
$$


If $S$ and $S^{\prime}$ are two compact subsets of $\mathbb{R}^{N}$ with $\mathcal{C}^{2}$ boundary such that $S \subset S^{\prime}$, then we note that $E\left(S^{\prime}\right) \subset E(S)$.

For any bounded open subset $\Omega$ of $\mathbb{R}^{N}$ with $S \subset \subset \Omega$ we define the functional $J_{h}: E(S) \rightarrow \mathbb{R}$ by setting

$$
J_{h}^{S}(\Omega, u)=\int_{\mathbb{R}^{N} \backslash S}\left(|\nabla u|^{2}+\mathbf{1}_{\{u>0\}}\left(1+\frac{1}{h} d_{\Omega}^{s}\right)_{+}\right),
$$

where $d_{\Omega}^{s}$ is the signed distance to $\Omega$ defined by $\left[5, \mathbf{1}_{A}\right.$ denotes the indicator function of any set $A \subset \mathbb{R}^{N}$ and $r_{+}=r \vee 0$ for any $r \in \mathbb{R}$. We write $J_{h}(\Omega, u)$ if there is no ambiguity about $S$.

Let us recall some existence and regularity results given in [3]:

Proposition 4.1 (Alt and Caffarelli [3]) Let $\Omega$ be an open subset of $\mathbb{R}^{N}$ such that $\Omega \backslash S$ is bounded and with $S \subset \subset \Omega$. Then there is at least a minimizer $u \in E(S)$ to $J_{h}(\Omega, \cdot)$. Moreover, $u$ is Lipschitz continuous and is harmonic in $\{u>0\} \backslash S$. Finally, $\mathcal{H}^{N-1}(\partial\{u>0\})<+\infty$.

REMARK 4.1 We note that $S \subset \subset\{u>0\}$ because $u$ is Lipschitz continuous with $u=1$ in $S$.

The existence of $u$ and its Lipschitz continuity come from [3, Theorem 1.3 and Corollary 3.3]. The fact that $u$ has a compact support is established in [3, Lemma 2.8], and its harmonicity in [3. Lemma 2.4]. The finiteness of $\mathcal{H}^{N-1}(\partial\{u>0\})$ is given in [3, Theorem 4.5].

We are now ready to define the discrete motions.

Let $\Omega_{0} \supset \supset S$ be a fixed initial condition. We define by induction the sequence $\left(\Omega_{n}^{h}\right)$ of open bounded subsets of $\mathbb{R}^{N}$ with $\Omega_{n}^{h} \supset \supset S$ by setting

$$
\Omega_{0}^{h}:=\Omega_{0} \quad \text { and } \quad \Omega_{n+1}^{h}:=\left\{u_{n}>0\right\} \cup\left\{x \in \Omega_{n}^{h}: d_{\partial \Omega_{n}^{h}}(x)>h\right\},
$$

where

$$
u_{n} \in \underset{v \in E(S)}{\operatorname{argmin}} J_{h}^{S}\left(\Omega_{n}^{h}, v\right)
$$

We call such a family of open sets a discrete motion. Of course, it is defined so that it converges to a solution of the front propagation problem (1) (see Theorem 5.2 and Remark 4.2).

In order to investigate the behavior of discrete motions, we need some properties of the minimizers of $J_{h}$.

Lemma 4.2 Let $\Omega$ and $u$ be as in Proposition 4.1 Let $\Omega^{\prime}=\{u>0\} \cup \hat{\Omega}_{h}$, where

$$
\hat{\Omega}_{h}:=\left\{y \in \Omega: d_{\partial \Omega}(y)>h\right\}=\left\{y \in \mathbb{R}^{N}: d_{\Omega}^{s}(y)<-h\right\} .
$$

Then $\left|\partial \Omega^{\prime}\right|=0$ and $u$ is the capacity potential of $\overline{\Omega^{\prime}}$.

REMARK 4.2 We do not claim that $u$ is positive in $\Omega^{\prime}$. For instance, consider a set $\Omega$ with two connected components $\Omega_{1}$ and $\Omega_{2}$ such that $S \subset \subset \Omega_{1}$. In this case, $u \equiv 0$ in $\Omega_{2}$. Notice that this explains why we define $\Omega_{n+1}^{h}:=\left\{u_{n}>0\right\} \cup\left\{x \in \Omega_{n}^{h}: d_{\partial \Omega_{n}^{h}}(x)>h\right\}$. Adding the set $\left\{x \in \Omega_{n}^{h}: d_{\partial \Omega_{n}^{h}}(x)>h\right\}$ prevents the discrete motion from a sudden disappearance of a connected component. Indeed, the discrete motion is built in order to approach a solution of the front propagation problem (1), and a connected component which does not contain any part of the source is expected to move with constant normal velocity -1 . 
Proof of Lemma 4.2 First notice that $\left|\partial \Omega^{\prime}\right|=0$. Indeed, we already know that $|\partial\{u>0\}|=0$ (because its $\mathcal{H}^{N-1}$-measure is finite from Proposition 4.1 . On the other hand, $\partial \hat{\Omega}_{h} \subset\{y \in \Omega$ : $d_{\partial \Omega^{\prime}}(y)=h$ \} also has a finite $\mathcal{H}^{N-1}$-measure thanks to [4, Lemma 2.4].

Let now $\epsilon>0$ be fixed and set, for any $\alpha>0, \Omega_{\alpha}=\left\{y \in \mathbb{R}^{N}: d_{\Omega^{\prime}}(y)<\alpha\right\}$. The set $\Omega_{\alpha}$ is open, bounded and satisfies $\Omega^{\prime} \subset \subset \Omega_{\alpha}$. Moreover, since $\mathbf{1}_{\Omega_{\alpha}} \rightarrow \mathbf{1}_{\overline{\Omega^{\prime}}}$ and $\Omega^{\prime}$ is bounded with $\left|\partial \Omega^{\prime}\right|=0$, for $\alpha>0$ enough small we have

$$
\int_{\Omega_{\alpha} \backslash \Omega^{\prime}}\left(1+\frac{1}{h} d_{\partial \Omega}^{s}\right)_{+} \leqslant \epsilon
$$

Let $v$ be the capacity potential of $\Omega_{\alpha}$ and set

$$
v_{k}(x)=v(x)+\frac{1}{k} d_{\mathbb{R}^{N} \backslash \Omega_{\alpha}}(x) \quad \forall x \in \mathbb{R}^{N}
$$

Then $\left(v_{k}\right)$ converges to $v$ in $H^{1}\left(\mathbb{R}^{N}\right)$ and $\left|\Omega_{\alpha} \backslash\left\{v_{k}>0\right\}\right|=0$. Therefore

$$
\begin{aligned}
J_{h}\left(\Omega, v_{k}\right) & =\int_{\mathbb{R}^{N} \backslash S}\left(\left|\nabla v_{k}\right|^{2}+\mathbf{1}_{\left\{v_{k}>0\right\}}\left(1+\frac{1}{h} d_{\Omega}^{s}\right)_{+}\right) \\
& \rightarrow \operatorname{cap}\left(\Omega_{\alpha}\right)+\int_{\mathbb{R}^{N} \backslash S} \mathbf{1}_{\Omega_{\alpha}}\left(1+\frac{1}{h} d_{\Omega}^{s}\right)_{+} .
\end{aligned}
$$

Since $J_{h}\left(\Omega, v_{k}\right) \geqslant J_{h}(\Omega, u)$, from (11) we get

$$
\begin{aligned}
\operatorname{cap}\left(\overline{\Omega^{\prime}}\right) & \geqslant \operatorname{cap}\left(\Omega_{\alpha}\right) \geqslant \lim _{k} J_{h}\left(\Omega, v_{k}\right)-\int_{\mathbb{R}^{N} \backslash S} \mathbf{1}_{\Omega_{\alpha}}\left(1+\frac{1}{h} d_{\Omega}^{s}\right)_{+} \\
& \geqslant J_{h}(\Omega, u)-\int_{\mathbb{R}^{N} \backslash S} \mathbf{1}_{\Omega_{\alpha}}\left(1+\frac{1}{h} d_{\Omega}^{s}\right)_{+} \geqslant \int_{\mathbb{R}^{N} \backslash S}\left(|\nabla u|^{2}-\mathbf{1}_{\Omega_{\alpha} \backslash\{u>0\}}\left(1+\frac{1}{h} d_{\Omega}^{s}\right)_{+}\right) \\
& \geqslant \int_{\mathbb{R}^{N} \backslash S}\left(|\nabla u|^{2}-\mathbf{1}_{\Omega_{\alpha} \backslash \Omega^{\prime}}\left(1+\frac{1}{h} d_{\Omega}^{s}\right)_{+}\right) \geqslant \int_{\mathbb{R}^{N} \backslash S}|\nabla u|^{2}-\epsilon .
\end{aligned}
$$

Thus $\int_{\mathbb{R}^{N} \backslash S}|\nabla u|^{2} \leqslant \operatorname{cap}\left(\overline{\Omega^{\prime}}\right)$, so $u$ is the capacity potential of $\overline{\Omega^{\prime}}$ by Lemma 3.3

Next we need to compare solutions to $J_{h}(\Omega, \cdot)$ for different $S$ and $\Omega$.

Proposition 4.3 Let $S_{1}$ and $S_{2}$ be the closures of two open bounded subsets of $\mathbb{R}^{N}$ with $\mathcal{C}^{2}$ boundary, and let $\Omega_{1}$ and $\Omega_{2}$ be open bounded subsets of $\mathbb{R}^{N}$ such that $S_{1} \subset \subset \Omega_{1}$ and $S_{2} \subset \subset \Omega_{2}$. Let $u_{1}$ and $u_{2}$ be, respectively, minimizers of $J_{h}^{S_{1}}\left(\Omega_{1}, \cdot\right)$ and $J_{h}^{S_{2}}\left(\Omega_{2}, \cdot\right)$. If $S_{1} \subset S_{2}$ and $\Omega_{1} \subset \Omega_{2}$, then $u_{1} \wedge u_{2}$ and $u_{1} \vee u_{2}$ are, respectively, minimizers of $J_{h}^{S_{1}}\left(\Omega_{1}, \cdot\right)$ and $J_{h}^{S_{2}}\left(\Omega_{2}, \cdot\right)$.

REMARK 4.3 1. In particular, if $J_{h}^{S_{2}}\left(\Omega_{2}, \cdot\right)$ has a unique minimizer $u_{2}$, then $\left\{u_{1}>0\right\} \subset\left\{u_{2}>0\right\}$.

2. This proposition still holds true if we replace, for $i=1,2, \Omega_{i}, S_{i}$ bounded by $\Omega_{i} \backslash S_{i}$ bounded; see Remark 3.1 and Lemma 4.5 
Proof of Proposition 4.3. Let us set

$$
\begin{aligned}
I:= & J_{h}^{S_{1}}\left(\Omega_{1}, u_{1} \wedge u_{2}\right)+J_{h}^{S_{2}}\left(\Omega_{2}, u_{1} \vee u_{2}\right)-J_{h}^{S_{1}}\left(\Omega_{1}, u_{1}\right)-J_{h}^{S_{2}}\left(\Omega_{2}, u_{2}\right) \\
= & \int_{\mathbb{R}^{N} \backslash S_{1}}\left(\left|\nabla\left(u_{1} \wedge u_{2}\right)\right|^{2}-\left|\nabla u_{1}\right|^{2}+\left(\mathbf{1}_{\left\{u_{1} \wedge u_{2}>0\right\}}-\mathbf{1}_{\left\{u_{1}>0\right\}}\right)\left(1+\frac{1}{h} d_{\Omega_{1}}^{s}\right)_{+}\right) \\
& +\int_{\mathbb{R}^{N} \backslash S_{2}}\left(\left|\nabla\left(u_{1} \vee u_{2}\right)\right|^{2}-\left|\nabla u_{2}\right|^{2}+\left(\mathbf{1}_{\left\{u_{1} \vee u_{2}>0\right\}}-\mathbf{1}_{\left\{u_{2}>0\right\}}\right)\left(1+\frac{1}{h} d_{\Omega_{2}}^{s}\right)_{+}\right) .
\end{aligned}
$$

Since $\Omega_{1} \subset \Omega_{2}$ we have $d_{\Omega_{2}}^{s} \leqslant d_{\Omega_{1}}^{s}$ in $\mathbb{R}^{N}$. It follows that

$$
\begin{aligned}
I \leqslant & \int_{\mathbb{R}^{N} \backslash S_{2}}\left(\left|\nabla\left(u_{1} \wedge u_{2}\right)\right|^{2}-\left|\nabla u_{1}\right|^{2}+\left|\nabla\left(u_{1} \vee u_{2}\right)\right|^{2}-\left|\nabla u_{2}\right|^{2}\right) \\
& +\int_{S_{2} \backslash S_{1}}\left(\left|\nabla\left(u_{1} \wedge u_{2}\right)\right|^{2}-\left|\nabla u_{1}\right|^{2}\right) \\
& +\int_{\mathbb{R}^{N} \backslash S_{1}}\left(\mathbf{1}_{\left\{u_{1} \wedge u_{2}>0\right\}}-\mathbf{1}_{\left\{u_{1}>0\right\}}\right)\left(1+\frac{1}{h} d_{\Omega_{1}}^{s}\right)_{+} \\
& +\int_{\mathbb{R}^{N} \backslash S_{2}}\left(\mathbf{1}_{\left\{u_{1} \vee u_{2}>0\right\}}-\mathbf{1}_{\left\{u_{2}>0\right\}}\right)\left(1+\frac{1}{h} d_{\Omega_{1}}^{s}\right)_{+}
\end{aligned}
$$

Moreover, by classical results,

$$
\left|\nabla\left(u_{1} \wedge u_{2}\right)\right|^{2}+\left|\nabla\left(u_{1} \vee u_{2}\right)\right|^{2}=\left|\nabla u_{1}\right|^{2}+\left|\nabla u_{2}\right|^{2} \text { a.e. in } \mathbb{R}^{N} .
$$

So we get

$$
I \leqslant \int_{S_{2} \backslash S_{1}}\left(\left|\nabla\left(u_{1} \wedge u_{2}\right)\right|^{2}-\left|\nabla u_{1}\right|^{2}+\left(\mathbf{1}_{\left\{u_{1} \wedge u_{2}>0\right\}}-\mathbf{1}_{\left\{u_{1}>0\right\}}\right)\left(1+\frac{1}{h} d_{\Omega_{1}}^{s}\right)_{+}\right) .
$$

But $u_{1} \wedge u_{2}=u_{1}$ on $S_{2} \backslash S_{1}$, which gives $I \leqslant 0$, and thus

$$
J_{h}^{S_{1}}\left(\Omega_{1}, u_{1} \wedge u_{2}\right)+J_{h}^{S_{2}}\left(\Omega_{2}, u_{1} \vee u_{2}\right) \leqslant J_{h}^{S_{1}}\left(\Omega_{1}, u_{1}\right)+J_{h}^{S_{2}}\left(\Omega_{2}, u_{2}\right) .
$$

Since $u_{1}$ and $u_{2}$ are minimizers we have

$$
J_{h}^{S_{1}}\left(\Omega_{1}, u_{1}\right) \leqslant J_{h}^{S_{1}}\left(\Omega_{1}, u_{1} \wedge u_{2}\right) \quad \text { and } \quad J_{h}^{S_{2}}\left(\Omega_{2}, u_{2}\right) \leqslant J_{h}^{S_{2}}\left(\Omega_{2}, u_{1} \vee u_{2}\right) .
$$

The inequalities in (13) and (14) are therefore equalities. Hence $u_{1} \wedge u_{2}$ and $u_{1} \vee u_{2}$ are respectively minimizers of $J_{h}^{S_{1}}\left(\Omega_{1}, \cdot\right)$ and $J_{h}^{S_{2}}\left(\Omega_{2}, \cdot\right)$.

We define the energy $\mathcal{E}(\bar{\Omega})$ by

$$
\mathcal{E}(\bar{\Omega})=|\Omega|+\operatorname{cap}(\bar{\Omega})
$$

(cf. (4)).

LEMMA 4.4 Let $\left(\Omega_{n}^{h}\right)$ be a discrete motion with $\left|\partial \Omega_{0}^{h}\right|=0$. Then the energy $\mathcal{E}\left(\overline{\Omega_{n}^{h}}\right)$ is nonincreasing with respect to $n$. More precisely,

$$
\mathcal{E}\left(\overline{\Omega_{n+1}^{h}}\right)-\mathcal{E}\left(\overline{\Omega_{n}^{h}}\right) \leqslant \int_{\mathbb{R}^{N}}\left(\mathbf{1}_{\Omega_{n}^{h} \backslash\left\{d_{\partial \Omega_{n}^{s}}^{s}<-h\right\}}-\mathbf{1}_{\left\{u_{n}>0\right\} \backslash\left\{d_{\partial \Omega_{n}^{h}}^{s}<-h\right\}}\right) \frac{1}{h} d_{\Omega_{n}^{h}}^{s} \leqslant 0,
$$

where $u_{n}$ is a minimizer for $J_{h}\left(\Omega_{n}^{h}, \cdot\right)$. 
Proof. Let us fix $n$. In order to simplify the notations, let us set

$$
\Omega:=\Omega_{n}^{h}, \quad \hat{\Omega}_{h}:=\left\{x \in \Omega: d_{\Omega}^{s}(x)<-h\right\}=\left\{x \in \Omega: d_{\partial \Omega}(x)>h\right\} .
$$

Let $u_{0}$ be the capacity potential of $\bar{\Omega}$ and $u$ be a minimizer of $J_{h}(\Omega, \cdot)$. We finally set $\Omega^{\prime}:=$ $\Omega_{n+1}^{h}=\{u>0\} \cup \hat{\Omega}_{h}$. Recall that $\Omega^{\prime} \in \mathcal{D}$ and $\left|\partial \Omega^{\prime}\right|=0$ : indeed, this is true for $n=0$ from the assumption and by Lemma 4.2 for $n \geqslant 1$. With these notations we have to prove that

$$
\mathcal{E}\left(\overline{\Omega^{\prime}}\right) \leqslant \mathcal{E}(\bar{\Omega}) .
$$

For this we introduce for any $k \geqslant 1$ the function $u_{k}$ defined by

$$
u_{k}(x)= \begin{cases}u_{0}(x)+\frac{1}{k} d_{\partial \Omega}(x) & \text { if } x \in \Omega \\ u_{0}(x) & \text { otherwise }\end{cases}
$$

Then $\left(u_{k}\right)$ converges to $u_{0}$ in $H^{1}\left(\mathbb{R}^{N}\right)$ and $\left\{u_{k}>0\right\}=\Omega$ a.e. because $\left\{u_{0}>0\right\} \subset \bar{\Omega}$ and $|\partial \Omega|=0$. Hence

$$
\begin{aligned}
\lim _{k} J_{h}\left(\Omega, u_{k}\right) & =\lim _{k} \int_{\mathbb{R}^{N} \backslash S}\left(\left|\nabla u_{k}\right|^{2}+\mathbf{1}_{\left\{u_{k}>0\right\}}\left(1+\frac{1}{h} d_{\Omega}^{s}\right)_{+}\right) \\
& =\operatorname{cap}(\bar{\Omega})+\int_{\mathbb{R}^{N} \backslash S} \mathbf{1}_{\Omega}\left(1+\frac{1}{h} d_{\Omega}^{s}\right)_{+} \\
& =\mathcal{E}(\bar{\Omega})-|\Omega|+\int_{\Omega \backslash \hat{\Omega}_{h}}\left(1+\frac{1}{h} d_{\Omega}^{s}\right)=\mathcal{E}(\bar{\Omega})+\int_{\Omega \backslash \hat{\Omega}_{h}} \frac{1}{h} d_{\Omega}^{s}-\left|\hat{\Omega}_{h}\right|
\end{aligned}
$$

On the other hand, since $\operatorname{cap}\left(\overline{\Omega^{\prime}}\right)=\int_{\mathbb{R}^{N} \backslash S}|\nabla u|^{2}$ from Lemma 4.2 and since $\left|\overline{\Omega^{\prime}}\right|=\left|\Omega^{\prime}\right|$, we also have

$$
\begin{aligned}
J_{h}(\Omega, u) & =\int_{\mathbb{R}^{N} \backslash S}\left(|\nabla u|^{2}+\mathbf{1}_{\{u>0\}}\left(1+\frac{1}{h} d_{\Omega}^{s}\right)_{+}\right) \\
& =\mathcal{E}\left(\overline{\Omega^{\prime}}\right)-\left|\Omega^{\prime}\right|+\int_{\{u>0\} \backslash \hat{\Omega}_{h}}\left(1+\frac{1}{h} d_{\Omega}^{s}\right)=\mathcal{E}\left(\overline{\Omega^{\prime}}\right)+\int_{\{u>0\} \backslash \hat{\Omega}_{h}} \frac{1}{h} d_{\Omega}^{s}-\left|\hat{\Omega}_{h}\right| .
\end{aligned}
$$

Noting that $J_{h}(\Omega, u) \leqslant J_{h}\left(\Omega, u_{k}\right)$, we get the desired claim.

Next we show that the solution does not blow up when $h$ becomes small.

Lemma 4.5 Let $R>0$ and $r_{0} \in\left(0, R / 2^{1 /(N-2)}\right)$ be fixed. Let us also fix $M$ such that $\sqrt{1+M} \geqslant$ $4(N-2) / r_{0}$. Then there is some $h_{0}=h_{0}\left(N, r_{0}, R, M\right)$ such that, for any $h \in\left(0, h_{0}\right)$ and $r \in$ $\left(r_{0}, R / 2^{1 /(N-2)}\right)$, for any $\Omega \in \mathcal{D}$ open bounded, for any $x \notin \bar{\Omega}$ with $r \leqslant d_{\Omega}(x), R \leqslant d_{S}(x)$ and for any $u$ minimizer of $J_{h}(\Omega, \cdot)$, we have

$$
d_{\{u>0\} \cup \hat{\Omega}_{h}}(x) \geqslant r-M h,
$$

where $\hat{\Omega}_{h}$ is defined by 10 .

Proof. The idea is to compare the solution with radial ones. For simplicity we assume that $N \geqslant 3$, the computation in the case $N=2$ being similar. We also suppose without loss of generality that $x=0$. 
Let us first investigate the problem of minimizing $J_{h}^{B_{R}^{c}}\left(B_{r}^{c}, \cdot\right)$, where $B_{r}=B(0, r)$ and $B_{R}=$ $B(0, R)$. Notice that neither the source $B_{R}^{c}$ nor the subset $B_{r}^{c}$ is bounded, but $B_{r}^{c} \backslash B_{R}^{c}=B_{R} \backslash B_{r}$ is bounded, so the previous results on the minimization problem apply (see Remark 3.1). Standard symmetrization arguments show that a minimizer $v$ of $J_{h}^{B_{R}^{c}}\left(B_{r}^{c}, \cdot\right)$ must be radially symmetric. For $\rho \in(0, R)$, let us denote by $v_{\rho}$ the (radial) harmonic function which vanishes on $\partial B_{\rho}$ and is equal to 1 on $\partial B_{R}$. We also set $J_{h}(\rho):=J_{h}^{B_{R}^{c}}\left(B_{r}^{c}, v_{\rho}\right)$. Notice that a minimizer of $J_{h}^{B_{R}^{c}}\left(B_{r}^{c}, \cdot\right)$ has to be either of the form $v_{\rho}$ with $\rho$ a minimizer of $J_{h}(\cdot)$, or constant equal to $v_{0}:=1$. Let us fix $h_{0}$ small enough that $r+h<R$ for $h \in\left(0, h_{0}\right)$. We have

$$
J_{h}\left(0^{+}\right)=J_{h}^{B_{R}^{c}}\left(B_{r}^{c}, v_{0}\right)=\frac{\alpha_{N-1}(r+h)^{N+1}}{h N(N+1)},
$$

where $\alpha_{N-1}$ is the volume of the unit sphere of $\mathbb{R}^{N}$. For $J_{h}(\rho)$ with $\rho>0$, we distinguish two cases. If $r+h<\rho<R$, then

$$
J_{h}(\rho)=\frac{\alpha_{N-1}(N-2)}{\rho^{2-N}-R^{2-N}} .
$$

If $0<\rho \leqslant r+h$, then

$$
\frac{J_{h}(\rho)}{\alpha_{N-1}}=\frac{N-2}{\rho^{2-N}-R^{2-N}}+\frac{1}{h}\left(\frac{(r+h)^{N+1}}{N(N+1)}+\frac{\rho^{N+1}}{N+1}-\frac{(r+h) \rho^{N}}{N}\right) .
$$

We show that $v_{0}$ cannot be a minimizer by comparing $J_{h}\left(0^{+}\right)$with $J_{h}(\rho)$ for $0<\rho \leqslant r+h$. Choosing $\rho=\beta \sqrt{h}$ with $\beta>0$, we have

$$
\begin{aligned}
\frac{1}{\alpha_{N-1}}\left(J_{h}(\rho)-J_{h}(0)\right. & =\rho^{N-2}\left(\frac{N-2}{1-(\rho / R)^{N-2}}+\frac{\rho^{3}}{h(N+1)}-\frac{(r+h) \rho^{2}}{h N}\right) \\
& \leqslant \rho^{N-2}\left(\frac{N-2}{1-\beta^{N-2} h^{(N-2) / 2} / R^{N-2}}+\frac{\beta^{3} h^{1 / 2}}{N+1}-\frac{r \beta^{2}}{N}\right) .
\end{aligned}
$$

Recalling that $r_{0} \in\left(0, R / 2^{1 /(N-2)}\right)$ is fixed, we choose

$$
\beta>\frac{N(2(N-2)+1)}{r_{0}}
$$

and then $h_{0}=h_{0}\left(N, \beta, r_{0}, R\right)>0$ small enough that

$$
1-\frac{\beta^{N-2} h_{0}^{(N-2) / 2}}{R^{N-2}}>\frac{1}{2} \text { and } \quad \frac{\beta^{3} h_{0}^{1 / 2}}{N+1}<1
$$

For all $h \in\left(0, h_{0}\right)$, we see that 15 is negative, which proves that $v_{0}$ is not a minimizer.

Therefore minimizers have to be of the form $v_{\rho}$ for some $\rho \in(0, R)$. On $(r+h, R), J_{h}(\rho)$ is increasing. For $\rho \in(0, r+h)$, we have

$$
\frac{J_{h}^{\prime}(\rho)}{\alpha_{N-1}}=\frac{(N-2)^{2} \rho^{1-N}}{\left(\rho^{2-N}-R^{2-N}\right)^{2}}+\frac{\rho^{N}}{h}-\frac{(r+h) \rho^{N-1}}{h} .
$$


The stationary points of $J_{h}$ on $(0, r+h]$ satisfy

$$
f(\rho):=\frac{(N-2)^{2}}{\left[\rho\left(1-(\rho / R)^{N-2}\right)\right]^{2}}-\frac{1}{h}(r+h-\rho)=0 .
$$

Notice that $\rho \mapsto f(\rho)$ is convex on $(0, r+h]$ and tends to $+\infty$ as $\rho \rightarrow 0^{+}$and as $\rho \rightarrow R^{-}$. If we find some value $\rho$ for which $f(\rho)$ is negative, then there are exactly two solutions to (18).

For this, let us choose $\rho=\beta \sqrt{h}$ with $\beta>0$. Then

$$
h f(\beta \sqrt{h})=\frac{(N-2)^{2}}{\left.\beta\left(1-\left(\beta h^{1 / 2} / R\right)^{N-2}\right)^{N-2}\right)^{2}}-r-h+\beta h^{1 / 2} .
$$

Choosing $\beta>0$ satisfying $[16]$ and

$$
\beta>4(N-2) / r_{0}^{1 / 2}
$$

and $h_{0}$ satisfying 17 and

$$
\beta h_{0}^{1 / 2}<r_{0} / 2
$$

we find that $f(\beta \sqrt{h})<0$ for $h \in\left(0, h_{0}\right)$.

Let us fix $h \in\left(0, h_{0}\right)$ and let $\rho_{1}$ and $\rho_{2}$ be respectively the smallest and largest solutions to 18. By the above arguments, $\rho_{1} \leqslant \beta \sqrt{h} \leqslant \rho_{2}$, where $\beta$ is defined as above. Since $J_{h}^{\prime}(\rho)=$ $\alpha_{N-1} \rho^{N-1} f(\rho)$, we have

$$
\begin{aligned}
J_{h}^{\prime \prime}\left(\rho_{1}\right) & =\alpha_{N-1} \rho_{1}^{N-1} f^{\prime}\left(\rho_{1}\right) \\
& =\alpha_{N-1} \rho_{1}^{N-1}\left[\frac{-2(N-2)^{2}\left(1-(N-1)\left(\rho_{1} / R\right)^{N-2}\right)}{\left(\rho_{1}\left(1-\left(\rho_{1} / R\right)^{N-2}\right)\right)^{3}}+\frac{1}{h}\right] \\
& \leqslant \alpha_{N-1} \rho_{1}^{N-1}\left[-\frac{2(N-2)^{2}}{\rho_{1}^{3}}\left(1-(N-1)\left(\frac{\rho_{1}}{R}\right)^{N-2}\right)+\frac{\beta^{2}}{\rho_{1}^{2}}\right] .
\end{aligned}
$$

If we choose $h_{0}>0$ satisfying [17], [19] and furthermore

$$
(N-1)\left(\frac{\beta h_{0}^{1 / 2}}{R}\right)^{N-2}<\frac{1}{2} \text { and } h_{0}^{1 / 2}<\frac{N-2}{\beta^{3}},
$$

we deduce that $J_{h}^{\prime \prime}\left(\rho_{1}\right)<0$ for $h \in\left(0, h_{0}\right)$ and $\rho_{1}$ is not a minimum to $J_{h}$. Therefore, $J_{h}$ is increasing on $\left(0, \rho_{1}\right)$, decreasing on $\left(\rho_{1}, \rho_{2}\right)$ and increasing on $\left(\rho_{2}, R\right)$. The minimum is achieved at $\rho=\rho_{2}$.

Let us now estimate $\rho_{2}$. We suppose that $h_{0}$ satisfies $[17$, , 19], 20] and

$$
h_{0} \leqslant \frac{r_{0}}{2 M} \quad \text { where } \quad 1+M \geqslant \frac{16(N-2)^{2}}{r_{0}^{2}} \text {. }
$$

Then, for all $h \in\left(0, h_{0}\right)$ and $r \in\left(r_{0}, R / 2^{1 /(N-2)}\right)$, we have $r-M h \geqslant r_{0} / 2>0$ and we compute

$$
\begin{aligned}
f(r-M h) & =\frac{(N-2)^{2}}{(r-M h)^{2}\left(1-((r-M h) / R)^{N-2}\right)^{2}}-(1+M) \\
& \leqslant \frac{4(N-2)^{2}}{(r-M h)^{2}}-(1+M) \leqslant 0 .
\end{aligned}
$$

Therefore $\rho_{2} \geqslant r-M h$. 
To summarize, we know that, setting $h_{0}=h_{0}\left(N, r_{0}, R, M\right)$ small enough, for all $h \in\left(0, h_{0}\right)$ and $r \in\left(r_{0}, R / 2^{1 /(N-2)}\right)$, the problem of minimizing $J_{h}^{B_{R}^{c}}\left(B_{r}^{c}, \cdot\right)$ has a unique solution $v_{\rho_{2}}$, which is radially symmetric and such that $\rho_{2} \geqslant r-M h$.

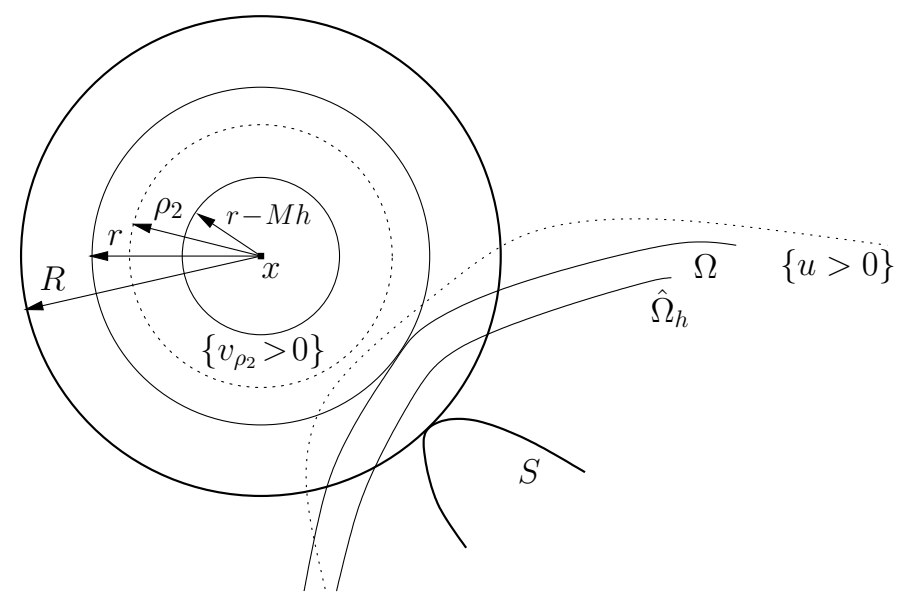

FIG. 1. Illustration of the proof of Lemma 4.5

Let now $\Omega \in \mathcal{D}, x \notin \bar{\Omega}$ with $R \leqslant d_{S}(x), r \leqslant d_{\Omega}(x)$, and let $u$ be a minimizer of $J_{h}(\Omega, \cdot)$. Since $S \subset B_{R}^{c}(x)$ and $\Omega \subset B_{r}^{c}(x)$, Proposition 4.3 states that $\{u>0\} \subset\left\{v_{\rho_{2}}>0\right\} \subset B_{r-M h}^{c}(x)$ (see Figure 11. Since $\hat{\Omega}_{h} \subset \Omega \subset B_{r}^{c}$, we conclude that $d_{\{u>0\} \cup \hat{\Omega}_{h}}(x) \geqslant r-M h$.

Finally, we show that the set $\{u>0\}$ satisfies some inequalities in the viscosity sense. Here again the regularity results of Alt and Caffarelli [3] play a crucial role. Let $\Sigma$ be an open set with $\mathcal{C}^{1,1}$ boundary such that $S \subset \subset \Sigma$ and $\Sigma \backslash S$ is bounded. We denote by $u_{S}^{\Sigma}$ the (classical) solution to 3 , (replacing $\Omega$ by $\Sigma$ ), i.e., the capacity potential of $\Sigma$ with respect to $S$.

LEMMA 4.6 Let $\Omega$ be a bounded open subset of $\mathbb{R}^{N}$ with $S \subset \subset \Omega$ and $u$ be a minimizer of $J_{h}(\Omega, \cdot)$. Set

$$
\hat{\Omega}_{h}=\left\{x \in \Omega: d_{\partial \Omega}(x)>h\right\} \quad \text { and } \quad \Omega^{\prime}=\{u>0\} \cup \Omega_{h} .
$$

Let $\Sigma$ be an open bounded subset of $\mathbb{R}^{N}$ with $\mathcal{C}^{1,1}$ boundary.

1. [Outward estimate] Suppose that $\Sigma$ is such that

$$
\{u>0\} \subset \Sigma \text { and } \exists x \in \partial \Sigma \cap \partial\{u>0\} .
$$

Then

$$
\left|\nabla u_{S}^{\Sigma}(x)\right| \geqslant\left(1+\frac{1}{h} d_{\Omega}^{S}(x)\right)_{+}^{1 / 2}
$$

2. [Inward estimate] Now assume that $\Sigma$ is such that

$$
S \subset \subset \Sigma, \quad \Sigma \subset \Omega^{\prime} \text { and } \exists x \in \partial \Sigma \cap \partial \Omega^{\prime} .
$$


Then

$$
\left|\nabla u_{S}^{\Sigma}(x)\right| \leqslant\left(1+\frac{1}{h} d_{\Omega}^{S}(x)\right)_{+}^{1 / 2} .
$$

Proof. Let us set $g_{\Omega}(x)=\left(1+d_{\Omega}^{s}(x) / h\right)_{+}$. We first prove the outward estimate. From [3, Lemma 4.10] we have

$$
\limsup _{x^{\prime} \rightarrow x, x^{\prime} \in\{u>0\}} \frac{u\left(x^{\prime}\right)}{d_{B}\left(x^{\prime}\right)} \geqslant \sqrt{g_{\Omega}(x)}
$$

for any ball $B$ contained in $\{u=0\}$ and tangent to $\{u>0\}$ at $x$. Let $v$ be the outward unit normal to $\Sigma$ at $x$, and $r>0$ be such that the ball $B:=B(x+r v, r)$ is tangent to $\Sigma$ at $x$. Then $B$ is also tangent to $\{u>0\}$ at $x$. Since by the maximum principle, $u \leqslant u_{S}^{\Sigma}$, we have

$$
\left|\nabla u_{S}^{\Sigma}(x)\right|=\limsup _{x^{\prime} \rightarrow x, x^{\prime} \in\{u>0\}} \frac{u_{S}^{\Sigma}\left(x^{\prime}\right)}{d_{B}\left(x^{\prime}\right)} \geqslant \limsup _{x^{\prime} \rightarrow x, x^{\prime} \in\{u>0\}} \frac{u\left(x^{\prime}\right)}{d_{B}\left(x^{\prime}\right)} \geqslant \sqrt{g_{\Omega}(x)} .
$$

We now turn to the proof of the inward estimate. We first prove that $u_{S}^{\Sigma} \leqslant u$ in $\left\{u_{S}^{\Sigma}>0\right\}$. Indeed, from Lemma 4.2, $u$ is the capacity potential of $\overline{\Omega^{\prime}}$. In particular, $u$ is harmonic in $\Omega^{\prime} \backslash S \supset \Sigma \backslash S$, $u=u_{S}^{\Sigma}$ on $\partial S$ and $0=u_{S}^{\Sigma} \leqslant u$ on $\partial\left\{u_{S}^{\Sigma}>0\right\}$. Hence $u_{S}^{\Sigma} \leqslant u$ in $\left\{u_{S}^{\Sigma}>0\right\}$. Let us note that $u=0$ on $\partial \Omega^{\prime}$. Therefore $u(x)=u_{S}^{\Sigma}(x)=0$.

We now consider two cases. If $x \notin \partial\{u>0\}$, then $x \in \partial \hat{\Omega}_{h}$; thus $d_{\Omega}^{s}(x)=-h$ and $g_{\Omega}(x)=0$. But $0 \leqslant u_{S}^{\Sigma} \leqslant u=0$ in a neighborhood of $x$ so that $\nabla u_{S}^{\Sigma}(x)=0$. Therefore

$$
\left|\nabla u_{S}^{\Sigma}(x)\right|=0=g_{\Omega}(x) .
$$

Let us now consider the case $x \in \partial\{u>0\}$. Then [3, Theorem 6.3] states that

$$
\sup _{B(x, r)}|\nabla u| \leqslant \sqrt{g_{\Omega}(x)}+m(r),
$$

where $m(r) \rightarrow 0$ as $r \rightarrow 0^{+}$. Since we want to prove that $\left|\nabla u_{S}^{\Sigma}(x)\right| \leqslant \sqrt{g_{\Omega}(x)}$, we can assume without loss of generality that $\nabla u_{S}^{\Sigma}(x) \neq 0$. Let $v$ be the outward unit normal to $\Sigma$ at $x$. Since $v=-\nabla u_{S}^{\Sigma}(x) /\left|\nabla u_{S}^{\Sigma}(x)\right|$, for $r>0$ sufficiently small, the segment $] x, x-r v$ [ is contained in $\Sigma$ and in $\left\{u_{S}^{\Sigma}>0\right\}$, and thus in $\{u>0\}$. So $u$ is smooth at each point of this segment. Since moreover $u \geqslant u_{S}^{\Sigma}$, we have, for some $\xi \in(x, x-r v)$,

$$
u_{S}^{\Sigma}(x-r v) \leqslant u(x-r v)=u(x)+\langle\nabla u(\xi),-r v\rangle \leqslant r\left(\sqrt{g_{\Omega}(x)}+m(r)\right) .
$$

Therefore

$$
\left|\nabla u_{S}^{\Sigma}(x)\right|=\lim _{r \rightarrow 0^{+}} \frac{u_{S}^{\Sigma}(x-r v)}{r} \leqslant \sqrt{g_{\Omega}(x)} .
$$

\section{Discrete motions and viscosity solutions}

Let us fix $\Omega_{0}$ open and bounded such that $S \subset \subset \Omega_{0}$. Let $\left(\Omega_{n}^{h}\right)_{n}$ be a discrete motion with $\Omega_{0}^{h}=\Omega_{0}$.

Let us now introduce a lower and upper envelope for the sequences $\left(\Omega_{n}^{h}\right)_{n}$ as the time step $h$ tends to $0^{+}$: the upper envelope $\mathcal{K}^{*}$ is

$$
\mathcal{K}^{*}(t):=\left\{x \in \mathbb{R}^{N}: \begin{array}{l}
\exists h_{k} \rightarrow 0^{+}, n_{k} \rightarrow+\infty, x_{k} \in \Omega_{n_{k}}^{h_{k}} \\
\text { with } x_{k} \rightarrow x \text { and } h_{k} n_{k} \rightarrow t
\end{array}\right\},
$$


while the lower envelope $\mathcal{K}_{*}$ is defined by its complement:

$$
\mathbb{R}^{N} \backslash \mathcal{K}_{*}(t)=\left\{x \in \mathbb{R}^{N}: \begin{array}{l}
\exists h_{k} \rightarrow 0^{+}, n_{k} \rightarrow+\infty, x_{k} \notin \Omega_{n_{k}}^{h_{k}} \\
\text { with } x_{k} \rightarrow x \text { and } h_{k} n_{k} \rightarrow t
\end{array}\right\} .
$$

LEMmA 5.1 The set $\mathcal{K}^{*}$ is closed while $\mathcal{K}_{*}$ is open. Moreover, the maps $t \mapsto \mathcal{K}^{*}(t)$ and $t \mapsto \widehat{\mathcal{K}}_{*}(t)$ are left lower semicontinuous on $(0,+\infty)$.

Proof. The fact that $\mathcal{K}^{*}$ is closed comes from its construction since the upper limit of sets is always closed. The argument works in a symmetric way for $\mathcal{K}_{*}$.

We now prove that $t \mapsto \mathcal{K}^{*}(t)$ is left lower semicontinuous on $(0,+\infty)$ (see Section 2 for the definition). We proceed by contradiction supposing there exist $t>0, x \in \mathcal{K}^{*}(t), \rho>0$ and a sequence $t_{p} \rightarrow t^{-}$such that $B(x, \rho) \cap \mathcal{K}^{*}\left(t_{p}\right)=\emptyset$. Therefore $d_{\mathcal{K}^{*}\left(t_{p}\right)}(x) \geqslant \rho>0$ for all $p$.

Set $R=d_{S}(x)$ and choose $r_{0}<\min \left\{\rho, R / 2^{1 /(N-2)}\right\}$ and $M>0$ with $\sqrt{1+M} \geqslant 8(N-2) / r_{0}$. Then Lemma 4.5 states that there is some $h_{0}=h_{0}\left(N, r_{0}, R, M\right)$ with the following property: for any $r \in\left(r_{0} / 2, R / 2^{1 / N-2}\right)$ and $h \in\left(0, h_{0}\right)$, for any $\Omega$ with $r \leqslant d_{\Omega}(x)$ and for any minimizer $u$ of $J_{h}(\Omega, \cdot)$, we have $d_{\{u>0\} \cup \hat{\Omega}_{h}}(x) \geqslant r-M h$, where $\hat{\Omega}_{h}=\left\{d_{\Omega}^{s}<-h\right\}$.

For $h \in\left(0, h_{0}\right)$, let $n_{h}=\left[t_{p} / h\right]$ be the integer part of $t_{p} / h$. From the definition of $\mathcal{K}^{*}\left(t_{p}\right)$ and $r_{0}$, we can find some $h_{1} \in\left(0, h_{0}\right)$ such that $d_{\Omega_{n_{h}}^{h}}(x) \geqslant r_{0}$ for any $h \in\left(0, h_{1}\right)$. We are going to prove by induction that

$$
d_{\Omega_{n_{h}+k h}^{h}}(x) \geqslant r_{0}-M k h \quad \text { for all } k \in\left\{0, \ldots, k_{0}^{h}\right\},
$$

where $k_{0}^{h}=\left[r_{0} /(2 M h)\right]$. Indeed, inequality 23 holds for $k=0$. Assume that it holds for some $k<k_{0}^{h}$. Let $u$ be a minimizer for $J_{h}\left(\Omega_{n_{h}+k h}^{h}, \cdot\right)$ and define

$$
\Omega_{n_{h}+(k+1) h}^{h}=\{u>0\} \cup\left\{y \in \Omega_{n_{h}+k h}^{h}: d_{\Omega_{n_{h}+k h}^{h}}(y)>h\right\} .
$$

Then since $r_{0}-M k h \geqslant r_{0} / 2$ and $r_{0}-M k h \leqslant r_{0} \leqslant R / 2^{1 /(N-2)}$, Lemma 4.5 recalled above implies that

$$
d_{\Omega_{n_{h}+(k+1) h}^{h}}(x) \geqslant r_{0}-M k h-M h .
$$

So $[23$ is proved.

Let us set $\tau=r_{0} /(4 M)$ and fix $s \in(0, \tau)$. Let $\left(k_{h}\right)$ be such that $k_{h} h \rightarrow s$ as $h \rightarrow 0^{+}$. We notice that $k_{h} \in\left\{0, \ldots, k_{0}^{h}\right\}$ for $h$ sufficiently small. Letting $h \rightarrow 0^{+}$in inequality 23 for any such $\left(k_{h}\right)$ implies that

$$
d_{\mathcal{K} *\left(t_{p}+s\right)}(x) \geqslant r_{0}-M s \geqslant r_{0} / 2>0 .
$$

Since $\tau$ does not depend on $x$ and $t_{p}$ and since $t_{p} \rightarrow t^{-}$, for $p$ large enough, we have $s=$ $t-t_{p} \leqslant \tau$. Therefore, from (24), we obtain $d_{\mathcal{K}^{*}(t)}(x)=d_{\mathcal{K}^{*}\left(t_{p}+s\right)}(x) \geqslant r_{0} / 2>0$, which contradicts the assumption $x \in \mathcal{K}^{*}(t)$.

The proof of the left lower semicontinuity of $\widehat{\mathcal{K}}_{*}$ is simpler. As above, we proceed by contradiction supposing that there exists $x \in \widehat{\mathcal{K}}_{*}(t)$ for $t>0$ and a sequence $t_{p} \rightarrow t^{-}$with $d_{\widehat{\mathcal{K}}_{*}\left(t_{p}\right)}(x) \geqslant \rho>0$ for all $p$. From the definition of $\Omega_{n_{h}+1}^{h}$, for $\left(n_{h}\right)$ such that $n_{h} h \rightarrow t_{p}$ and $h$ sufficiently small, we have $B_{\rho / 2}(x) \subset \Omega_{n_{h}}^{h}$. From the definition of $\Omega_{n_{h}+1}^{h}$, we have therefore

$$
B_{\rho / 2-h}(x) \subset\left\{y \in \Omega_{n_{h}}^{h}: d_{\partial \Omega_{n_{h}}^{h}}(x)>h\right\} \subset \Omega_{n_{h}+1}^{h} .
$$


By induction we prove in a similar way that, for any $k \leqslant \rho /(4 h)$,

$$
B_{\rho / 2-k h}(x) \subset\left\{y \in \Omega_{n_{h}+(k-1) h}^{h}: d_{\partial \Omega_{n_{h}+(k-1) h}^{h}}(x)>h\right\} \subset \Omega_{n_{h}+k}^{h} .
$$

Letting now $h \rightarrow 0^{+}$we get

$$
B_{\rho / 4}(x) \cap \widehat{\mathcal{K}}_{*}\left(t_{p}+s\right)=\emptyset \quad \text { for all } s \in[0, \rho / 4] .
$$

Since $\rho$ is independent of $p$, we get a contradiction by taking $p$ so large that $t-t_{p}=s \leqslant \rho / 4$.

THEOREM 5.2 The tube $\mathcal{K}^{*}$ (respectively $\mathcal{K}_{*}$ ) is a viscosity subsolution (respectively supersolution) to the front propagation problem $V=h(x, \Omega)$, where

$$
h(x, \Omega)=-1+\bar{h}(x, \Omega)
$$

and $\bar{h}$ is defined by 2 . .

Proof. Let us set $\Omega^{h}:=\bigcup_{n}\{n h\} \times \Omega_{n}^{h}$. Let $\left(t_{0}, x_{0}\right) \in \mathcal{K}^{*}$ with $t_{0}>0$ be such that there is a smooth regular tube $\mathcal{K}_{r}$ with $\mathcal{K}^{*} \subset \mathcal{K}_{r}$ and $x_{0} \in \partial \mathcal{K}_{r}\left(t_{0}\right)$. Without loss of generality we can assume that $\mathcal{K}^{*} \cap \partial \mathcal{K}_{r}=\left\{\left(t_{0}, x_{0}\right)\right\}$. Then by standard stability arguments (see [7]), one can find a sequence of smooth regular tubes $\mathcal{K}_{r}^{k}$ converging to $\mathcal{K}_{r}$ in the $\mathcal{C}^{1, \mathrm{~b}}$ sense (see Section 2 for the definition), and sequences $h_{k} \rightarrow 0$ and $n_{k} \rightarrow+\infty$ such that $\Omega^{h_{k}} \subset \mathcal{K}_{r}^{k},\left(n_{k} h_{k}, x_{k}\right) \rightarrow\left(t_{0}, x_{0}\right), x_{k} \in \partial \Omega_{n_{k}}^{h_{k}}$ and $x_{k} \in \partial \mathcal{K}_{r}^{k}\left(n_{k} h_{k}\right)$.

Let $u$ be a minimizer of $J_{h_{k}}\left(\Omega_{n_{k}-1}^{h_{k}}, \cdot\right)$. By definition of the discrete motion, we have

$$
\Omega_{n_{k}}^{h_{k}}=\{u>0\} \cup\left\{y \in \Omega_{n_{k}-1}^{h_{k}}: d_{\Omega_{n_{k}-1}^{h_{k}}}^{S}(y)<-h_{k}\right\} .
$$

Let $v_{k}:=u_{S}^{\mathcal{K}_{r}^{k}\left(n_{k} h_{k}\right)}$ be the capacity potential of $\mathcal{K}_{r}^{k}\left(n_{k} h_{k}\right)$.

Let us first assume that $x_{k} \in \partial\{u>0\}$ for some subsequence of $\left(x_{k}\right)$ (still denoted by $\left(x_{k}\right)$ ). The case $x_{k} \in \operatorname{int}\{u=0\}$ for any $k$ is treated later. From the discrete viscosity condition in Lemma 4.6 and the inclusion $\Omega_{n_{k}-1}^{h_{k}} \subset \mathcal{K}_{r}^{k}\left(\left(n_{k}-1\right) h_{k}\right)$, we know that

$$
\left|\nabla v_{k}\left(x_{k}\right)\right| \geqslant\left(1+\frac{1}{h_{k}} d_{\Omega_{n_{k}-1}^{h_{k}}}^{s}\left(x_{k}\right)\right)_{+}^{1 / 2} \geqslant\left(1+\frac{1}{h_{k}} d_{\mathcal{K}_{r}^{k}\left(\left(n_{k}-1\right) h_{k}\right)}^{s}\left(x_{k}\right)\right)_{+}^{1 / 2}
$$

Hence

$$
\frac{1}{h_{k}} d_{\mathcal{K}_{r}^{k}\left(\left(n_{k}-1\right) h_{k}\right)}^{S}\left(x_{k}\right) \leqslant-1+\left|\nabla v_{k}\left(x_{k}\right)\right|^{2} .
$$

Let us now recall that the normal velocity of $\mathcal{K}_{r}^{k}$ at a point $(t, x) \in \partial \mathcal{K}_{r}^{k}$ is given by $-\frac{\partial}{\partial t} d_{\mathcal{K}_{r}^{k}(t)}^{s}(x)$. Since $x_{k} \in \partial \mathcal{K}_{r}^{k}\left(n_{k} h_{k}\right)$, since $\left(n_{k} h_{k}, x_{k}\right) \rightarrow\left(t_{0}, x_{0}\right)$ and since $\mathcal{K}_{r}^{k}$ converges to $\mathcal{K}_{r}$, we have

$$
d_{\mathcal{K}_{r}^{k}\left(\left(n_{k}-1\right) h_{k}\right)}^{s}\left(x_{k}\right)=d_{\mathcal{K}_{r}^{k}\left(n_{k} h_{k}\right)}^{s}\left(x_{k}\right)-h_{k} \frac{\partial}{\partial t} d_{\mathcal{K}_{r}^{k}\left(n_{k} h_{k}\right)}^{s}\left(x_{k}\right)+h_{k} \epsilon(k)=h_{k} V_{\left(t_{0}, x_{0}\right)}^{\mathcal{K}_{r}}+h_{k} \epsilon(k),
$$

where $\epsilon(k) \rightarrow 0$ as $k \rightarrow+\infty$ and $V_{\left(t_{0}, x_{0}\right)}^{\mathcal{K}_{r}}$ is the normal velocity of $\mathcal{K}_{r}$ at $\left(t_{0}, x_{0}\right)$. From 26 we get, for $k$ large enough,

$$
h\left(x_{k}, \mathcal{K}_{r}^{k}\left(n_{k} h_{k}\right)\right)=-1+\left|\nabla v_{k}\left(x_{k}\right)\right|^{2} \geqslant V_{\left(t_{0}, x_{0}\right)}^{\mathcal{K}_{r}}+\epsilon(k)
$$


Letting $k \rightarrow+\infty$, we obtain

$$
h\left(x_{0}, \mathcal{K}_{r}\left(t_{0}\right)\right)=\lim _{k} h\left(x_{k}, \mathcal{K}_{r}^{k}\left(n_{k} h_{k}\right)\right) \geqslant V_{\left(t_{0}, x_{0}\right)}^{\mathcal{K}_{r}} .
$$

The above equality is a straightforward application of [13, Theorem 8.33] since $\mathcal{K}_{r}^{k}$ converges to $\mathcal{K}_{r}$ in the $\mathcal{C}^{1, \mathrm{~b}}$ sense (see Section 2 for the definition).

We now assume that $x_{k} \in \operatorname{int}\{u=0\}$ for any $k$. Then from 25] we have

$$
d_{\Omega_{n_{k}-1}^{h_{k}}}^{s}\left(x_{k}\right)=-d_{\partial \Omega_{n_{k}-1}^{h_{k}}}\left(x_{k}\right)=-h_{k} .
$$

Arguing as above we get

$$
-h_{k}=d_{\Omega_{n_{k}-1}^{h_{k}}}^{s}\left(x_{k}\right) \geqslant d_{\mathcal{K}_{r}^{k}\left(\left(n_{k}-1\right) h_{k}\right)}^{s}\left(x_{k}\right)=h_{k} V_{\left(t_{0}, x_{0}\right)}^{\mathcal{K}_{r}}+h_{k} \epsilon(k),
$$

where $\epsilon(k) \rightarrow 0$. Dividing by $h_{k}$ and letting $k \rightarrow+\infty$ gives

$$
V_{\left(t_{0}, x_{0}\right)}^{\mathcal{K}_{r}} \leqslant-1 \leqslant-1+\left|\nabla u_{S}^{\mathcal{K}_{r}\left(t_{0}\right)}\left(x_{0}\right)\right|^{2}=h\left(x_{0}, \mathcal{K}_{r}\left(t_{0}\right)\right) .
$$

So we have finally proved that $\mathcal{K}^{*}$ is a subsolution.

We now show that $\mathcal{K}_{*}$ is a supersolution. The proof starts exactly as above: if there is a smooth regular tube $\mathcal{K}_{r}$ with $\mathcal{K}_{r} \subset \mathcal{K}_{*}$ and some $\left(t_{0}, x_{0}\right) \in \partial \mathcal{K}_{*}$ with $t_{0}>0$ and $x_{0} \in \partial \mathcal{K}_{r}\left(t_{0}\right)$, then one can find a sequence of smooth regular tubes $\mathcal{K}_{r}^{k}$ converging to $\mathcal{K}_{r}$ in the $\mathcal{C}^{1, \mathrm{~b}}$ sense and sequences $h_{k} \rightarrow 0$ and $n_{k} \rightarrow+\infty$ such that $\mathcal{K}_{r}^{k}\left(n h_{k}\right) \subset \Omega_{n}^{h_{k}}$ for any $n,\left(n_{k} h_{k}, x_{k}\right) \rightarrow\left(t_{0}, x_{0}\right)$, and $x_{k} \in$ $\partial \Omega^{h_{k}}\left(n_{k} h_{k}\right) \cap \partial \mathcal{K}_{r}^{k}\left(n_{k} h_{k}\right)$. Let $u$ be a minimizer of $J_{h_{k}}\left(\Omega_{n_{k}-1}^{h_{k}}, \cdot\right)$. Then 25 holds for $\Omega_{n_{k}}^{h_{k}}$.

Then using Lemma 4.6 we get

$$
\left|\nabla v_{k}\left(x_{k}\right)\right| \leqslant\left(1+\frac{1}{h_{k}} d_{\Omega_{n_{k}-1}^{h_{k}}}^{s}\left(x_{k}\right)\right)_{+}^{1 / 2} \leqslant\left(1+\frac{1}{h_{k}} d_{\mathcal{K}_{r}^{k}\left(\left(n_{k}-1\right) h_{k}\right)}\left(x_{k}\right)\right)_{+}^{1 / 2},
$$

where $v_{k}:=u_{S}^{\mathcal{K}_{r}^{k}\left(n_{k} h_{k}\right)}$ is the capacity potential of $\mathcal{K}_{r}^{k}\left(n_{k} h_{k}\right)$ with respect to $S$. Since $x_{k} \in$ $\partial \Omega^{h_{k}}\left(n_{k} h_{k}\right)$, we have from 25 that $d_{\Omega^{h_{k}\left(n_{k} h_{k}\right)}}\left(x_{k}\right) \geqslant-h_{k}$. Therefore inequality 27 can also be written as

$$
\frac{1}{h_{k}} d_{\mathcal{K}_{r}^{k}\left(\left(n_{k}-1\right) h_{k}\right)}\left(x_{k}\right) \geqslant-1+\left|\nabla v_{k}\left(x_{k}\right)\right|^{2} .
$$

As before we have

$$
d_{\mathcal{K}_{r}^{k}\left(\left(n_{k}-1\right) h_{k}\right)}^{S}\left(x_{k}\right)=h_{k} V_{\left(t_{0}, x_{0}\right)}^{\mathcal{K}_{r}}+h_{k} \epsilon(k)
$$

Hence

$$
h\left(x_{k}, \mathcal{K}_{r}^{k}\left(n_{k} h_{k}\right)\right)=-1+\left|\nabla v_{k}\left(x_{k}\right)\right|^{2} \leqslant V_{\left(t_{0}, x_{0}\right)}^{\mathcal{K}_{r}}+\epsilon(k) \rightarrow V_{\left(t_{0}, x_{0}\right)}^{\mathcal{K}_{r}} .
$$

Then we can complete the proof as above to get the required condition:

$$
h\left(x_{0}, \mathcal{K}_{r}\left(t_{0}\right)\right) \leqslant V_{\left(t_{0}, x_{0}\right)}^{\mathcal{K}_{r}} .
$$

In particular we immediately obtain the following theorem: 
THEOREM 5.3 Let $\Omega_{0}$ be an open bounded subset of $\mathbb{R}^{N}$ such that $S \subset \subset \Omega_{0}$. Let $\mathcal{K}^{+}$and $\mathcal{K}^{-}$be, respectively, the largest and smallest viscosity solutions to the front propagation problem (1) with initial position $\Omega_{0}$. Then

$$
\mathcal{K}^{-} \subset \mathcal{K}_{*} \subset \mathcal{K}^{*} \subset \mathcal{K}^{+}
$$

In particular, if the problem has a unique solution, i.e., $\overline{\mathcal{K}^{-}}=\mathcal{K}^{+}$, then

$$
\overline{\mathcal{K}^{-}}=\overline{\mathcal{K}}_{*}=\mathcal{K}^{*}=\mathcal{K}^{+} .
$$

Proof. Since $\mathcal{K}^{+}$contains any subsolution and $\mathcal{K}^{-}$is contained in any supersolution (see [8]), we have $\mathcal{K}^{*} \subset \mathcal{K}^{+}$and $\mathcal{K}^{-} \subset \mathcal{K}_{*}$. The inclusion $\mathcal{K}_{*} \subset \mathcal{K}^{*}$ holds by construction. Hence the result follows.

\section{The energy is non-increasing along the flow}

Let $\Omega_{0}$ be a bounded open subset of $\mathbb{R}^{N}$. In this section we assume that the front propagation problem (1) with initial position $\Omega_{0}$ satisfies

$$
\left|\partial \Omega_{0}\right|=0 \text { and }\left|\mathcal{K}^{+} \backslash \mathcal{K}^{-}\right|=0,
$$

where $\mathcal{K}^{+}$and $\mathcal{K}^{-}$denote the maximal and minimal solutions respectively.

REMARK 6.1 Assumption 28 - which is a strong uniqueness condition for the viscosity solution of the front propagation problem (1) - is not too restrictive. Indeed, it is generic in the following sense: let $\left(\Omega_{0}^{\lambda}\right)_{\lambda>0}$ be a strictly increasing family of bounded open initial positions containing the source, i.e.,

$$
\text { for all } 0<\lambda<\lambda^{\prime}, \quad S \subset \subset \Omega_{0}^{\lambda} \subset \subset \Omega_{0}^{\lambda^{\prime}} .
$$

If $\mathcal{K}_{\lambda}^{+}$(respectively $\mathcal{K}_{\lambda}^{-}$) is the maximal (respectively minimal) viscosity solution to 11 with initial position $\Omega_{0}^{\lambda}$, then 280 holds for all $\lambda>0$ except for a countable subset. See [8, Props. 4 and 5] for details. For simplicity of notation, we have chosen to consider the case $\lambda=1$ and to assume that (28) holds for the initial position $\Omega_{0}$.

THEOREM 6.1 Under assumption (28), there is a set $\mathcal{T} \subset[0,+\infty)$ of full measure such that

$$
\mathcal{E}\left(\mathcal{K}^{+}(t)\right) \leqslant \mathcal{E}\left(\mathcal{K}^{+}(s)\right) \quad \text { for all } s, t \in \mathcal{T}, s<t .
$$

Proof. Let $\left(\Omega_{n}^{h}\right)$ be a discrete motion starting from $\Omega_{0}$. Recall for later use that, from Lemma 4.4

$$
\mathcal{E}\left(\overline{\Omega_{n}^{h}}\right) \leqslant \mathcal{E}\left(\bar{\Omega}_{0}\right) \quad \forall n \geqslant 0, \forall h>0,
$$

because we have assumed that $\left|\partial \Omega_{0}\right|=0$. Let $\mathcal{K}^{*}$ and $\mathcal{K}_{*}$ be the associated generalized evolutions defined by (21) and (22). We have

$$
\mathcal{K}^{-} \subset \mathcal{K}_{*} \subset \mathcal{K}^{*} \subset \mathcal{K}^{+}
$$

Let

$$
\mathcal{T}:=\left\{t \in[0,+\infty):\left|\mathcal{K}^{+}(t) \backslash \mathcal{K}^{-}(t)\right|=0\right\} .
$$

From assumption 28] and the Fubini theorem, the set $\mathcal{T}$ is of full measure in $[0,+\infty)$. 
We first prove that

$$
\mathcal{E}\left(\mathcal{K}^{+}(t)\right) \leqslant \mathcal{E}\left(\bar{\Omega}_{0}\right) \quad \forall t \in \mathcal{T}
$$

For this, let $t \in \mathcal{T}$, and let $h_{k} \rightarrow 0^{+}$and $n_{k} \rightarrow+\infty$ be such that $h_{k} n_{k} \rightarrow t$. For simplicity we set $\Omega_{k}:=\Omega_{n_{k}}^{h_{k}}$. Since the Kuratowski upper limit of $\left(\Omega_{k}\right)$ is contained in $\mathcal{K}^{+}(t)$, which is a compact subset, the sequence $\left(\Omega_{k}\right)$ is bounded. Since moreover the upper limit of ( $\left.\mathbb{R}^{N} \backslash \Omega_{k}\right)$ is contained in $\mathbb{R}^{N} \backslash \mathcal{K}^{-}(t)$, the latter with boundary at a positive distance from $S$, there is some $r>0$ such that $S_{r} \subset \Omega_{k}$ for any $k$ sufficiently large (see (8) for the definition of $S_{r}$ ). Since finally the capacity is non-increasing with respect to the inclusion, Lemma 3.2 yields

$$
\liminf _{k} \operatorname{cap}\left(\bar{\Omega}_{k}\right) \geqslant \operatorname{cap}\left(\mathcal{K}^{+}(t)\right) .
$$

The next step towards 30 amounts to showing that

$$
\left|\mathcal{K}^{+}(t)\right| \leqslant \liminf \left|\Omega_{k}\right|
$$

Let $R>0$ be so large that $\mathcal{K}^{+}(t) \subset \subset B_{R}$, where $B_{R}=B(0, R)$. By definition of the Kuratowski upper limit and the construction of $\mathcal{K}_{*}$, we have

$$
\mathbf{1}_{B_{R} \backslash \mathcal{K}_{*}(t)} \geqslant \underset{k}{\limsup } \mathbf{1}_{B_{R} \backslash \Omega_{k}} .
$$

The Fatou lemma then states that

$$
\left|B_{R} \backslash \mathcal{K}_{*}(t)\right| \geqslant \lim \sup \left|B_{R} \backslash \Omega_{k}\right|,
$$

whence 32 follows since $\mathcal{K}^{-}(t) \subset \mathcal{K}_{*}(t)$ and $\left|\mathcal{K}^{+}(t)\right|=\left|\mathcal{K}^{-}(t)\right|$ because $t \in \mathcal{T}$.

Combining (31), 32) and 29) finally gives

$$
\mathcal{E}\left(\mathcal{K}^{+}(t)\right) \leqslant \liminf _{k} \mathcal{E}\left(\bar{\Omega}_{k}\right) \leqslant \mathcal{E}\left(\bar{\Omega}_{0}\right) \quad \forall t \in \mathcal{T} .
$$

This proves 30 .

Let now $0 \leqslant s \leqslant t$ with $s, t \in \mathcal{T}$. From the uniqueness of the solution starting from $K_{0}$, the maximal solution to the front propagation problem starting at time $s$ from $\mathcal{K}^{+}(s)$ is equal at time $t$ to $\mathcal{K}^{+}(t)$. Since $\left|\partial \mathcal{K}^{+}(s)\right|=0$, because $s \in \mathcal{T}$, inequality 30 states that

$$
\mathcal{E}\left(\mathcal{K}^{+}(t)\right) \leqslant \mathcal{E}\left(\mathcal{K}^{+}(s)\right)
$$

which is the desired result.

\section{Acknowledgments}

We wish to thank Luis Caffarelli, Antonin Chambolle and Marc Dambrine for fruitful discussions. The authors are partially supported by the ACI grant JC 1041 "Mouvements d'interface avec termes non-locaux" from the French Ministry of Research. 


\section{REFERENCES}

1. Allaire, G., Jouve, F., \& Toader, A.-M. Structural optimization using sensitivity analysis and a level-set method. J. Comput. Phys. 194 (2004), 363-393. Zbl pre02056194 MR 2033390

2. Almgren, F., TAYlor, J., \& WANG, L. Curvature-driven flows: a variational approach. SIAM J. Control Optim. 31 (1993), 387-438. Zbl 0783.35002 MR 1205983

3. Alt, H. W., \& CAfFarelli, L. A. Existence and regularity for a minimum problem with free boundary. J. Reine Angew. Math. 325 (1981), 105-144. Zbl 0449.35105 MR 0618549

4. Alvarez, O., Cardaliaguet, P., \& Monneau, R. Existence and uniqueness for dislocation dynamics with nonnegative velocity. Interfaces Free Bound. 7 (2005), 415-434. Zbl 1099.35148 MR 2191694

5. Ambrosio, L. Minimizing movements. Rend. Accad. Naz. Sci. XL Mem. Mat. Appl. (5) 19 (1995), 191246. Zbl 0957.49029 MR 1387558

6. Cardaliaguet, P. On front propagation problems with nonlocal terms. Adv. Differential Equations 5 (2000), 213-268. Zbl 1029.53081 MR 1734542

7. Cardaliaguet, P. Front propagation problems with nonlocal terms. II. J. Math. Anal. Appl. 260 (2001), 572-601. Zbl 1002.35064 MR 1845570

8. Cardaliaguet, P., \& Ley, O. On some flows in shape optimization. Arch. Ration. Mech. Anal. 183 (2007), 21-58. MR 2259339

9. Chambolle, A. An algorithm for mean curvature motion. Interfaces Free Bound. 6 (2004), 195-218. Zbl 1061.35147 MR 2079603

10. Evans, L. C., \& Spruck, J. Motion of level sets by mean curvature. I. J. Differential Geom. 33 (1991), 635-681. Zbl 0726.53029 MR 1100206

11. Evans, L. C., \& Spruck, J. Motion of level sets by mean curvature. IV. J. Geom. Anal. 5 (1995), 77-114. Zbl 0829.53040 MR 1315658

12. Flucher, M., \& RUMPF, M. Bernoulli's free-boundary problem, qualitative theory and numerical approximation. J. Reine Angew. Math. 486 (1997), 165-204. Zbl 0909.35154 MR 1450755

13. Gilbarg, D., \& Trudinger, N. S. Elliptic Partial Differential Equations of Second Order. 2nd ed., Springer, Berlin (1983). Zbl 0562.35001 MR 0737190

14. Henrot, A., \& Pierre, M. Variation et optimisation de formes-Une analyse géométrique. Springer, Paris (2005). Zbl 1098.49001 